\title{
Analysis of Spatial Features in CBIR System
}

\author{
Minakshi Kaushik \\ Dept of CSE \\ IIMT Engineering College \\ Meerut India
}

\author{
Rahul Sharma \\ Dept of CSE \\ IIMT Engineering College \\ Meerut India
}

\author{
Ankit Vidhyarthi \\ Dept of CSE \\ Sunder Deep Engg. College \\ Ghaziabad India
}

\begin{abstract}
Content based image retrieval from large database has become an area of wide interest nowadays in many applications. Content-based image retrieval (CBIR) technique use image content to search and retrieve digital images. Content-based image retrieval (CBIR) is an important research area for manipulating large amount of image databases. In this paper the analysis work is done for finding the spatial features and collects them into a frame to view all the spatial features and the scope of implementing these features into the image retrieval. The commercial image search engines available as on date are: QBIC, VisualSeek, Virage, Netra, PicSOM, FIRE, AltaVista, etc. Region-Based Image Retrieval (RBIR) is a promising extension of CBIR.
\end{abstract}

The shape and spatial features are quite simple to derive and effective, and can be extracted in real time. Our analysis is able to propose a system that has the advantage of increasing the retrieval accuracy and decreasing the retrieval time.

\section{General Terms}

CBIR, Spatial features, Feature Extraction.

\section{Keywords}

Feature Vector, CBIR, Edge Histogram, Color, Texture

\section{INTRODUCTION}

CBIR is the sub problem of content based retrieval (CBR) it is necessary to develop power tools for retrieving images from the web where the number and size of digital image is growing fast. In addition, CBIR is also the key technology for improving the interface between user and computer.

CBIR is a technique used for retrieving similar images from image database. It uses various features of image to matching the query image to database images.

Content Based Image Retrieval (CBIR) is a technique which uses visual contents, normally called as features, such as shape, color, texture, edge, etc. to search images from large scale image databases according to users' requests in the form of a query image. Content based retrieval of visual data requires a paradigm that differs significantly from both traditional databases and text based image understanding systems. The challenge in CBIR is to develop the methods that will increase the retrieval accuracy and reduce the retrieval time. The CBIR technology has been used in several applications such as fingerprint identification, biodiversity information systems, digital libraries, crime prevention, medicine, historical research.

This paper is exploring the features of image which can be used for the similarity measurements. The work is done till the feature selection area. Our emphasis is to the exploring the maximum features and find the important relevant features, contributing in effective image retrieval.
Features can be classified mainly into 3 categories: spatial, spectral and frequency.

The main emphasis of this paper is to analysis of the spatial features. Section 2 will explore the Feature Extraction categories and sub categories of spatial features. Section 3 consist the result and section 4 states our conclusion and future work.

Content based image retrieval system consists of following modules:

1. Feature Extraction: In this module the features of interest are calculated for image database. This step is done in offline manner.

2. Feature extraction of query image: This module calculates the feature of the query image. Query image can be a part of image database or it may not be a part of image database. This is online calculation.

3. Similarity measure: This module compares the feature database of the existing images with the query image on basis of the similarity measure of the interest.

4. Retrieval and Result: This module will display the matching images to the user based on threshold value of similarity measure.

\section{FEATURE EXTRACTION}

Mapping the image pixels into the feature space is known as feature extraction. Extracted features are used to represent images for searching, indexing and browsing images in an image database. Feature extraction is a means of extracting compact but semantically valuable information from images. This information is used as a signature for the image. Similar images should have similar signatures. Feature extraction of the image in the database is typically conducted off-line so computation complexity is not a significant issue. This section introduces spatial features: texture, shape, and color, size and Edge density which are used most often to extract the features of an image.

\subsection{Color}

One of the most important features visually recognized by humans in images is color. Humans tend to distinguish images based mostly on color features. Because of this, color features are the most widely used in CBIR systems and the most studied in literature. Color is a powerful descriptor that simplifies object identification, and is one of the most frequently used visual features for content-based image retrieval. To extract the color features from the content of an image, a proper color space and an effective color descriptor have to be determined. 


\subsubsection{Color Histogram}

A histogram is the distribution of the number of pixels for an image. The number of elements in a histogram depends on the number of bits in each pixel of an image. If we consider a pixel depth of $n$ bit, $2^{n}-1$ and the histogram will have $2^{n}$ elements the histogram based image retrieval consists of two techniques: Global Color Histograms (GCH) and Local Color Histograms (LCH). The GCH represents images with single histogram. First the GCHs of database images are computed and stored them in a database. Then the GCH of the query image is computed. The Euclidian distance metrics is used to measure the similarity between the query image and the database images. In order to identify the relevant images a fixed threshold is used. In case of $\mathrm{LCH}$, images are divided into fixed blocks of size $8 \times 8$. For each block, its color histogram is obtained. The same procedure followed for $\mathrm{GCH}$ is applied for identifying the relevant images.

\subsubsection{Color Moments}

Images are differentiating by the measurement 'Color Moment' based on the color features. There are four color moments are defined:

$$
\text { Mean }=\sum_{i=1}^{n} \sum_{j=1}^{m} x_{i j} / m n
$$

Variance $=\frac{1}{n m} \sum_{i=1}^{n} \sum_{j=1}^{m}\left(X_{i j}-\text { mean }\right)^{2}$

Skewness $=\sqrt[3]{\frac{1}{n m}} \sum_{i=1}^{n} \sum_{j=1}^{m}\left(X_{i j}-\text { mean }\right)^{3}$

\section{Standard Deviation $=\sqrt{\text { var iance }}$}

Where $\mathrm{X}_{\mathrm{ij}}$ is the pixel value of the $\mathrm{i}^{\text {th }}$ row and $\mathrm{j}^{\text {th }}$ column

\subsubsection{Color coherent vector}

We define a color's coherence as the degree to which pixels of that color are members of large similarly-colored regions. We refer to these significant regions as coherent regions, and observe that they are of significant importance in characterizing images. Color histogram does not consider the spatial information of pixels. Color coherent vector addresses this problem. In CCV each histogram bin is partitioned into two types: coherent and incoherent. Pixel value belongs to a large informally colored region falls into coherent type. Otherwise it falls into incoherent type.

\subsection{Texture}

A variety of techniques has been used for measuring texture similarity; the best-established rely on comparing values of what are known as second-order statistics calculated from query and stored images. Essentially, these calculate the relative brightness of selected pairs of pixels from each image. From these it is possible to calculate measures of image texture such as the degree of contrast, coarseness, homogeneity and regularity [Tamura et al, 1978], or periodicity, correlation and entropy [Liu and Picard, 1996].Features derived from this approach include moments such as mean, standard deviation, average energy, entropy, skewness and kurtosis.

$$
\begin{aligned}
& \text { Contrast }=\sum_{\mathrm{i}} \sum_{\mathrm{j}=1}(\mathrm{i}-\mathrm{j})^{2} \mathrm{P}_{\mathrm{d}}(\mathbf{i}, \mathbf{j}) \\
& \text { Correlation }=\frac{\sum_{u=0}^{N} \sum_{v=0}^{N} I(u, v) I(u+x),(v+y)}{\sum_{u=0}^{N} \sum_{v=0}^{N} I^{2}(u, v)}
\end{aligned}
$$

Entropy $=\frac{1}{M} \sum_{x=1}^{M} \sum_{y=1}^{N} I_{i}(x, y)\left(-\operatorname{InI} I_{i}(x, y)\right)$

Energy $=\frac{1}{M} \sum_{x=1}^{M} \sum_{y=1}^{N} I_{i}^{2}(x, y)$

Homogeneity $=\sum_{i j} \sum_{j} \frac{P_{d}(i, j)}{1+|i-j|}$

Kurtosis $=\frac{\sum_{x=1}^{M} \sum_{y=1}^{N}\left(I_{i}(x, y)-\mu\right)^{4}}{M \times N \times \sigma^{4}}-3$

Mean $=\frac{\sum_{x=1}^{M} \sum_{y=1}^{N}\left(I_{i}(x, y)\right.}{M \times N}$
Skewness $=\frac{\sum_{x=1}^{M} \sum_{y=1}^{N}\left(I_{i}(x, y)-\mu\right)^{3}}{M \times N \times \sigma^{2}}$

Standard Deviation $=\sqrt{\frac{\sum_{x=1}^{M} \sum_{y=1}^{N}\left(I_{i}(x, y)-\mu\right)^{2}}{M \times N}}$

Variance $=\frac{1}{n m} \sum_{i=1}^{n} \sum_{j=1}^{m}\left(X_{i j}-\text { mean }\right)^{2}$ 
Tamura: Tamura et al took the approach of devising texture features that correspond to human visual perception.

Coarseness has a direct relationship to scale and repetition rates. The coarseness measure is then the average of $\operatorname{Sopt}(x, y)=$ $2 k$ opt over the picture.

\section{Coarseness}

$\mathrm{F}_{\mathrm{crs}}=\frac{1}{m \times n} \sum_{i=1}^{m} \sum_{j=1}^{n} S_{o p t}(i, j)$

Contrast aims to capture the dynamic range of grey levels in an image, together with the polarization of the distribution of black and white.

\section{Contrast}

$$
\mathrm{F}_{\mathrm{con}}=\frac{\sigma}{1 / 4}
$$

Where the kurtosis $\alpha 4=\mu 4 / \sigma 4, \mu 4$ is the fourth moment about the mean, and $\sigma 2$ is the variance

Directionality is a global property over a region. A histogram, $h d$, of edge probabilities is then built up by counting all points with magnitude greater than a threshold and quantizing by the edge angle. The histogram will reflect the degree of directionality.

$$
\mathrm{F}_{\mathrm{dir}}=\sum_{p \phi \in w_{p}}^{n_{p}} \sum_{w_{p}}\left(\phi-\phi_{p}\right)^{2} H_{D}(\phi)
$$

$$
\text { Smoothness }=R=1-1 /\left(1+\sigma_{2}(z)\right)
$$

\subsection{Shape}

Shape may be defined as the characteristic surface configuration of an object; an outline or contour. It permits an object to be distinguished from its surroundings by its outline [15].Two main types of shape feature are commonly used global features such as aspect ratio, circularity and moment invariants [Niblack et al, 1993] and local features such as sets of consecutive boundary segments [Mehrotra and Gary, 1995]. Aspect ratio is the ratio of the width and height of the shape.

\section{Aspect ratio $=$ Width: height}

\section{Circularity}

$f_{\text {circ }}=\frac{4 \pi A}{P^{2}}$

Invariant moments has 4 contour sequence moments which are

$$
\begin{aligned}
& \mathbf{F}_{1}=\frac{\left(M_{2}\right)^{\frac{1}{2}}}{m 1} ; \\
& \mathbf{F}_{2}=\frac{\left(M_{3}\right)}{\left(M_{2}\right)^{\frac{3}{2}}} \\
& \mathbf{F}_{3}=\frac{\left(M_{4}\right)}{\left(M_{2}\right)^{2}} \\
& \mathbf{F}_{4}=\frac{\left(M_{5}\right)}{\left(M_{2}\right)^{\frac{5}{2}}}
\end{aligned}
$$

Geometric moment invariants are

$$
\begin{aligned}
& \phi_{1}=\eta_{20}+\eta_{02} \\
& \phi_{2}=\left(\eta_{20}-\eta_{02}\right)^{2}+4 \eta_{11}^{2}
\end{aligned}
$$

$$
\begin{aligned}
& \phi_{3}=\left(\eta_{30}-3 \eta_{12}\right)^{2}+\left(3 \eta_{21}^{2}-\eta_{03}\right)^{2} \\
& \phi_{4}=\left(\eta_{30}-\eta_{12}\right)^{2}+\left(\eta_{21}+\eta_{03}\right)
\end{aligned}
$$

In Local features Gradient vector flow is another measurement. Gradient vector flow (GVF) is a static external force used in active contour method. The external force field is referred to as the GVF field. The GVF field $V(x, y)$ is a vector field given by

$$
F_{e x t}^{(p)}=V(x, y)
$$

$V(x, y)=[u(x, y), v(x, y)]$ that minimizes the energy functional

$$
\varepsilon=\iint \mu\left(\mu_{x}^{2}+\mu_{y}^{2}+v_{x}^{2}+v_{y}^{2}\right)+|\nabla f|^{2}|V-\nabla f|^{2} d x d y
$$

\subsection{Edge Density}

Edge Histogram Descriptor (EHD) captures the spatial distribution of edges like the color layout descriptor does by capturing colors. It is useful in image matching when the texture is not homogeneous.

\subsubsection{Edge histogram descriptor}

The edge histogram descriptor (EHD) represents the local edge distribution by dividing image space into $4 \times 4$ sub images and representing the local distribution of each sub image by a histogram. An Edge detector is applied to each of the fixed number of block, treating them as $2 \times 2$ pixel image. Pixel intensities for $2 \times 2$ blocks are computed by taking the average intensity values of the underlying pixel of the image block. Edge detector mask are shown in figure. 


\subsubsection{Vertical}

\begin{tabular}{|c|c|}
\hline 1 & -1 \\
\hline 1 & -1 \\
\hline
\end{tabular}

\subsubsection{Horizontal}

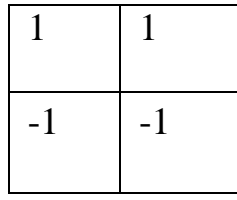

\subsubsection{3 $45^{\circ}$ Diagonal}

\begin{tabular}{|l|l|}
\hline$\sqrt{ } 2$ & 0 \\
\hline 0 & $-\sqrt{ } 2$ \\
\hline
\end{tabular}

\begin{tabular}{|l|l|}
\hline 0 & $\sqrt{ } 2$ \\
\hline$-\sqrt{ } 2$ & 0 \\
\hline
\end{tabular}

\begin{tabular}{|l|l|}
\hline 2 & -2 \\
\hline-2 & 2 \\
\hline
\end{tabular}

\subsubsection{Isotropic}

\subsection{Size}

The size of the images measures in dimensions. This is another feature by which we can extract the same size of images we can find the image by particular size.

\section{RESULT}

We selected the 300 images to create image database on the domain of archeology. In order to select the appropriate feature from the list of the features we apply the PCA (Principal Component Analysis) on the Query image. And get the Eigen values and cumulative variance percentage values. After reducing the feature set, we got the reduced feature set. These features are selected features, used in CBIR system to retrieve the image.

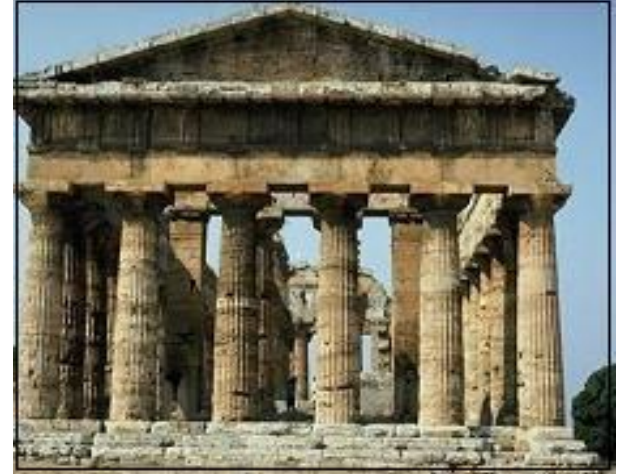

Fig 1: Query Image

\begin{tabular}{|c|c|c|c|}
\hline feature & no. eigen value & Fvariance & teurnulative Variance \\
\hline 1 & 64.133378 & 16.931412 & 16.931412 \\
\hline 19 & 51.314784 & 13.547263 & 30.478675 \\
\hline 2 & 51.000000 & 13.464159 & 43.942834 \\
\hline 16 & 50.670437 & 13.377153 & 57.319967 \\
\hline 17 & 34.086816 & 8.983186 & 66.303173 \\
\hline 19 & 27.029150 & 7.135780 & 73.438952 \\
\hline 15 & 24.667543 & 6.512308 & 79.951261 \\
\hline 4 & 18.649129 & 4.923428 & 84.874689 \\
\hline 3 & 16.830085 & 4.443406 & 09.316095 \\
\hline 13 & 16.400686 & 4.329832 & 93.647928 \\
\hline 10 & 12.639746 & 3.336932 & 96.984860 \\
\hline 12 & 6.349083 & 1.674858 & 98.659718 \\
\hline 5 & 2.429358 & 0.641094 & 99.300812 \\
\hline 6 & 1.000000 & 0.264003 & 99.564015 \\
\hline 7 & 0.968994 & 0.255817 & 99.820632 \\
\hline 31 & 0.300736 & 0.079395 & 99.900027 \\
\hline 20 & 0.165165 & 0.043604 & 99.943631 \\
\hline 19 & 0.066534 & 0.017565 & 99.961197 \\
\hline
\end{tabular}

Fig 2: Feature Values

\begin{tabular}{|c|c|c|}
\hline \multicolumn{3}{|r|}{ cursulat1ve Var 1abce (percent) } \\
\hline 1 & 64.133370 & 16.931412 \\
\hline 18 & 51.314784 & 30.478675 \\
\hline 8 & 51.000000 & 43.942834 \\
\hline 16 & 50.670437 & 57.319987 \\
\hline 17 & 39.026816 & 66.303173 \\
\hline 14 & 27.029150 & 73.438952 \\
\hline 15 & 24.667593 & 79.951261 \\
\hline 4 & 18.649129 & 84.874689 \\
\hline$\beta$ & 16.830885 & 89.318095 \\
\hline 13 & 16.400696 & 93.647928 \\
\hline 10 & 12.639746 & 96.984060 \\
\hline 12 & 6.344083 & 90.659716 \\
\hline$s$ & 2,428358 & 99.300812 \\
\hline
\end{tabular}

Fig 3: Reduced Set of Features

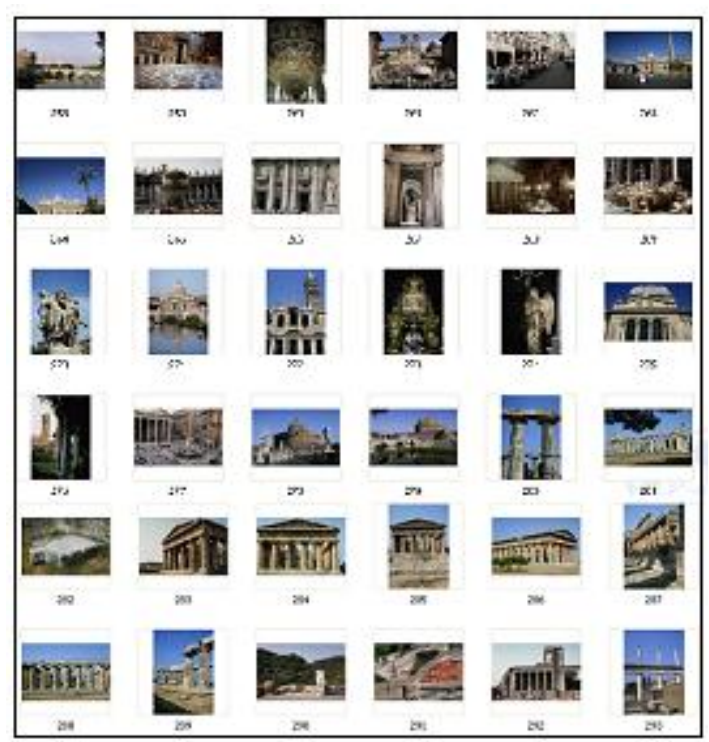

Fig 4: Snapshot of Image Database 


\section{CONCLUSION AND FUTURE WORK}

In this paper we have explored all the feature set in spatial domain of CBIR system. We have seen in previous work that the performance is based on color, texture only and combination of color and texture. In this analysis we found that the results of image retrieval using the individual feature color or texture are not as good as using combining these features. After combining the important sub features of color, texture and shape, the result is improved and the efficiency of image retrieval is better.PCA is used which helped in feature selection. The final result is reduced set of features which is the set of selected features. These reduced features can be used for image retrieval and give the better performance.

There are two other domains to extract the features. These domains are spectral and frequency domain. In future the features can be extracted using spectral and frequency domain and can extract the image by combining all the domains.

\section{REFERENCES}

[1] S.R. Kodituwakku "Comparison of Color Features for Image Retrieval", et al. / Indian Journal of Computer Science and Engineering Vol. 1 No. 3 207-211S.R

[2] Shriram K V, P.L.K.Priyadarsini, "CBIR - An analysis and suggestions for improvement”, International Journal of Computer Applications (0975 - 8887) Volume 42- No.14, March 2012

[3] Selvarajah and S.R. Kodituwakku (2011), "Analysis and Comparison of Texture Features for Content Based Image Retrieval." International Journal of Latest Trends in Computing (E-ISSN: 2045-5364) 108 Volume 2, Issue 1, March 2011

[4] P. B. Thawari \& N. J. Janwe (2011), "CBIR Based on color and texture", International Journal of Information Technology and Knowledge Management January-June 2011, Volume 4, No. 1, pp. 129-132

[5] Arnold W.M. Smeulders, Senior Member, IEEE, Marcel Worring, Simone Santini, Member, IEEE, Amarnath Gupta, Member, IEEE, and Ramesh Jain, Fellow,
IEEE(2000), "Content-Based Image Retrieval at the End of the Early Years", IEEE Transactions on pattern analysis and machine intelligence, VOL. 22, NO. 12, December 2000

[6] Hiremath P. S, Jagadeesh Pujari, "Content Based Image Retrieval using Color Boosted Salient Points and Shape features of an image."

[7] Shriram K V and P.L.K.Priyadarsini (2012), "CBIR - An analysis and suggestions for improvement", International Journal of Computer Applications (0975 - 8887) Volume 42- No.14, March 2012

[8] B.V. Ramana Reddy, A. Suresh, M. Radhika Mani3, and V.Vijaya Kumar(2009), "Classification of Textures Based on Features Extracted from Preprocessing Images on Random Windows", International Journal of Advanced Science and Technology Volume 9, August, 2009

[9] Noah Keen ,“Color Moments” February 10, 2005

[10] Greg Pass Ramin Zabih Justin Miller, "Comparing Images Using Color Coherence Vectors" Cornell University Ithaca, NY 14853

[11] P. S. Hiremath and Jagadeesh Pujari , " Content Based Image Retrieval based on Color, Texture and Shape features using Image and its complement"

[12] Vaibhav Gupta ,(2012), "Evolution of CBIR approaches for differently sized images" International Journal on Computer Science and Engineering (IJCSE), Vol. 4 No. 01 January 2012

[13] Pradnya Rane ,Pallavi Kulkarni, Suchita Patil and B.B.Meshram, "Feature based image retrieval of images for CBIR", IJCEM International Journal of Computational Engineering \& Management, Vol. 14, October 2011

[14] Swati V. Sakhare \& Vrushali G. Nasre, "Design of Feature Extraction in Content Based Image Retrieval (CBIR) using Color and Texture", International Journal of Computer Science \& Informatics, Volume-I, Issue-II, 2011

[15] Alaa Al-Hamami and Hisham Al-Rashdan(2010), "Improving the Effectiveness of the color coherence vector",The International Arab Journal of Information Technology, Vol. 7, No. 3, July 2010 